\title{
Epitaxial growth of vertically aligned piezoelectric diphenylalanine peptide microrods with uniform polarization
}

Vu Nguyen, Kory Jenkins, and Rusen Yang*

Department of Mechanical Engineering, University of Minnesota, Minneapolis, MN 55414, USA

*To whom correspondence should be addressed: yangr@umn.edu

\begin{abstract}
Energy harvesting with piezoelectric nanomaterials spurred the development of self-powered nanosystems, and piezoelectric biomaterials are expected to play an important role in the biomedical field. Bio-inspired piezoelectric diphenylalanine (FF) peptide microstructures were fabricated on various substrates through anovel epitaxial growth approach. The low-temperature process produced vertically aligned FF peptidemicrorods with hexagonally arranged nanochannels and uniform polarization. Direct measurement of the piezoelectricity was achieved for the first time from a solidFF peptide single crystal and yielded an effective piezoelectric coefficient $d_{33}$ at $9.9 \mathrm{pm} / \mathrm{V}$.The dense and aligned FF peptidemicrorods are advantageous for energy and sensing applications.
\end{abstract}

Key Words: Piezoelectricity; peptide;polarization; diphenylalanine; microrods; nanochannels

\section{Introduction}

Molecular self-assembly of bio-inspired materials has attracted much research effort in recent years due to its potential to fabricate novel bottom-up structures for new applications. Among them diphenylalanine (FF) peptide, which is highly biocompatible with low to no cytotoxicity [13], has been widely explored recently as a potential candidate for various applications such assupercapacitors[4-6], electromechanical sensing and actuation[7],optics[8, 9], nanostructure fabrication[10], and drug delivery [2]. Many of those applications aim to take advantage of the hydrophilicnanochannels and charge polarization which are inherent in thecrystal self-assembled 
by linear FF molecules[11, 12]. Strong piezoelectricity has been demonstrated in FF peptide nanotubes. A high effective piezoelectric coefficient $d_{15}$ at $60 \mathrm{pm} / \mathrm{V}$ was measured from the shear response of FF peptide hollow tubes [13] and $d_{33}$ was estimated in the range of 5-50 pm/V [13-15]. However,the current long and slender micro/nano tubular structures lacking in largescale alignment may prevent effective access to the nanochannels. In addition, the coercive field was estimated on the order of $\sim 30 \mathrm{MV} / \mathrm{cm}$ which made polarization switching practically impossible [15]. The current FF peptide nanotubes show random polarizations from the growth. The growth of FF peptide nano and microstructures with uniform polarizationis needed in order to observe and take advantage of the piezoelectricity at large scale.

Here we report a new and scalable approach to the controlled fabrication on various substrates of vertically aligned FF peptide microrods with uniform polarization. We start by engineering a textured seed layer withpreferential vertical orientation, and then grow the FF peptidemicrorods epitaxially from this seed layer. Unlike high temperature processes which yield cyclic-FF structure withoutnanochannels and charge polarization [16-18],our simple process is mostly done at room temperature and the producedlinear-FF peptide is of hexagonal crystal structure with nanochannels and spontaneous polarization. This novel approach provides for good alignment of the material in cases where it will be used for structural applications. In applications where large-scale, uniform piezoelectricity of the material is required, this can be achieved by the application of an electric field during growth. This polarization under electric field is independent of the good structural alignment achieved by our method.Characterization techniques such as X-ray Diffraction (XRD) and Piezoresponse Force Microscopy (PFM) consistently suggest that the hexagonal crystal structure is maintained. The measured effective piezoelectric coefficient $d_{33}$ of theFF peptidemicrorods is comparable to that of $\mathrm{ZnO}$ nanowires[19].Our work can enable new applications of bio-inspired materials in areas such as energy harvesting and storage, electromechanical sensing and actuation, drug delivery, as well as fundamental studies of FF-based structures.

\section{Experimental}

\subsection{Preparation of seed layer}

Diphenylalanine lyophilized powder H-Phe-Phe-OH (FF) was purchased from Bachem and stored in an enclosed dry container at $0^{\circ} \mathrm{C}$. 1,1,1,3,3,3-hexafluoro-2-propanol (HFP) was 
purchased from Sigma Aldrich and stored in a dry container under ambient conditions. The schematic of the fabrication process is shown in Figure 1a-c. First,the FF stock solution was prepared by dissolving lyophilized FF powderinHFP to a concentration of $50 \mathrm{mg} / \mathrm{mL}$ in a glove box (water content $<1000 \mathrm{ppm}$ ) to minimize water absorption to the source materials. $25 \mu \mathrm{L}$ of the stock solution was then dropped on a substrate placed in a desiccator. The drop was quickly vaporizedas the desiccator was pumped down to 200 Torr in 10 seconds and then vented with compressed dry air (water content $<6 \mathrm{ppm}$ ). A transparent amorphous film was formed on the substrate after the evaporation completed, Figure 1a.The low water vapor content in the growth environment with relative humidity $(\mathrm{RH})$ lower than $50 \%$ isessential to prevent unintentional crystallization of the amorphous film [20]. This amorphous film was then transferredto an enclosed box which was connected in a closed loop of flowingmoist air with $\mathrm{RH} 100 \%$ (Figure 1b).The amorphous film was intentionally crystallized into a seed layer by vigorously circulating the humid air through the enclosed box for 90 seconds.

\subsection{Epitaxial growth of FF peptide microrods}

The epitaxial growth from the seed layer was achieved by the precipitation of a saturated FF aqueous solution, Figure 1c. First a concentrated FF aqueous solution was prepared by mixing $75 \mathrm{mg} \mathrm{FF}$ with $50 \mathrm{~mL}$ deionized (DI) water and kept in an oven at $65^{\circ} \mathrm{C}$ until the FF powder was fully dissolved. The substrate with the prepared seed layer was placed floating upside down on the surface of concentrated FF solution right after the solution was taken from the oven. Ventilation from a small fan was used to facilitate cooling and evaporation of water in the concentrated FF solution. Most of thewater had evaporated after about 6 hours. The substrate with FF peptide microrods was then taken out, briefly cleaned with DI water, and dried with compressed air. The growth process is not limited by the substrate and FF peptide microrods have been successfully grown on silicon, indium tin oxide (ITO), platinum and gold (see Figure S1c-f). Samples on ITO-coated silicon substrate were used for the characterization demonstrated in this paper.

\subsection{Morphology and structure characterization}

Crystallized seed layers and as-grown FF peptidemicrorods were first examined using aNikon Eclipse LV150 optical microscope.All samples were coated with either gold or platinum and analyzed using afield emission JOEL 6500 scanning electron microscope. Both top view images 
and side view images were taken from theFF peptidemicrorods. The crystalline structure of both seed layers and FF peptidemicrorods were subject to XRD analysis after they were crushed into powders and pressed on a $<100>$ silicon substrate. XRD measurement were made using Bruker D8 system with Cobalt anode $(\lambda=1.78899 \AA)$.

\subsection{Piezoelectricity measurement}

Piezoelectricity measurements of the FF peptide seed layerandaligned microrods were conducted with an Asylum MFP 3D system. The probe used was an Asylum Research ASYELEC-01 with $\mathrm{Ti} / \mathrm{Ir}$ conductive coating, nominal stiffness of $2 \mathrm{~N} / \mathrm{m}$, and nominal resonance frequency of 70 $\mathrm{kHz}$. For characterization of effective piezoelectric coefficient $\mathrm{d}_{33}$, a small area $(0.5 \times 0.5 \mu \mathrm{m})$ was scanned in Single Frequency Mode with very low frequency (10kHz) to avoid contact resonance (typically around $300 \mathrm{kHz}$ ). Drive voltage amplitude on the tip varied from $2 \mathrm{~V}$ to $14 \mathrm{~V}$ and the corresponding vibration amplitude of the tip was recorded and averaged over the scanned small area to calculate $\mathrm{d}_{33}$.To record the phase map of the samples, Dual AC Resonance Tracking (DART) mode was used to enhance the vibration signal as well as to eliminate topography crosstalk on the resonance frequency for a large scan size $(90 \times 90 \mu \mathrm{m})$.

\section{Results and discussions}

Figure 1d and e show the cross section of the crystalline seed layer and FF microrods after 6hour room-temperature epitaxial growth in the absence of electric field. The vertical microrods are dense and were grown uniformly over the entire $1.25 \times 1.25 \mathrm{~cm}$ substrate(see Figure S1b for low magnification top view of the array).Their height is approximately $10 \mu \mathrm{m}$ from the seed layer, or $15 \mu \mathrm{m}$ from the substrate. The microrods were solid with the diameter mostly ranging from $10 \mu \mathrm{m}$ to $20 \mu \mathrm{m}$, which forms the largest reported solid microrod array to our best knowledge[9].No branches were observed in FF peptidemicrorods even after 12 hours of growth, as shown in Figure S1c-f. The growth of the microrods is the result of the self-assembly of FF molecules on the seed layer as the saturated FF aqueous solution evaporates. The microrod was expected to have a hexagonal crystal structure $[11,21]$. As shown in Figure 1f, the FF peptidemicrorod can be considered as a bundle of idealized close-packing of hydrophobic nanotubes with hydrophilic hollow channels. The channel is of a van der Waals' diameter of $\sim 10 \AA$ and filled with water molecules[21]. Six FF peptide molecules constitute the circumference and the translation of those molecules along the axial direction creates each tube. The side chains with inert phenyl 
group emanate from the channel cores and are exposed from the side wall. The more active carboxyl group and amine group forms a hydrogen bond and connects the FF peptide molecules along the axial direction.Thus, unlike the neutral phenyl groupson the wall, the positively charged amine groups or negatively charged carboxyl groups at the endof the nanochannel assist the self-assembly of FF peptide molecules for the fast growth along axial direction. Consequently, a highly textured seed layer with nanochannels preferentially orientated normal to the substrate is critical for the epitaxial growth of high quality FF peptide nano or microstructures with desired alignment.

The crystal structure of FF peptide seed layers and FF peptidemicrorods were confirmed with XRD analysis. Figure 2 shows typical XRD spectra of the seed layer and the FF peptidemicrorod. The microrod structure results in an almost identical XRD pattern to that of the seed layer. The hexagonal structure ( $P 6_{1}$ space group) proposed by Gorbitz and the resultant crystallographic data in the Cambridge Crystallographic Data Centre (CCDC) file number 163340 are in agreement with our XRD spectra[21]. The XRD data indicates the FF peptidecrystals are aclose-packing of nanotubes with a diameter of $24 \AA$. The hydrophilic nanochannels within FF hexagonal structures are deemed able to host different ions or molecules $[2,4,6]$ for various applications such as energy storage or drug delivery. Consequently, the vertical FF peptidemicrorod with well-aligned nanochannels can increase the accessibility and transport of foreign ions or molecules.

The highly textured and crystallized seed layer is critical for the successful epitaxial growth of FF peptidemicrorods, and we explore systematically the formation of FF seed layersshown in Figure 3. The scattered dark spots in Figure 3 are the dark ITO-coatedsilicon substrate revealed through vertical FF peptidedomainswhich are good waveguides with low longitudinal optical loss [9, 22], while the bright parts are mainly horizontal FF peptide strips.We found that both the thickness of the initial FF amorphous layer and the supply of water molecules during the crystallization control the orientation ofthe seed layer.The thickness of the amorphous layer is proportional to the concentration and the amount of FF-HFP stock solution dropped onto the substrate[18, 20] (also shown in Figure S2). Figure 3 shows the effect of the thickness of the amorphous layer and the supply of water molecules. $25 \mu \mathrm{LFF}$ solution of concentration either $50 \mathrm{mg} / \mathrm{mL}$ or $100 \mathrm{mg} / \mathrm{mL}$ was dropped and dried on a $1.25 \times 1.25 \mathrm{~cm}$ ITO-coated silicon substrate 
to form an amorphous FF layer.Crystallization of these layers was achieved with either stationary orcirculating moist air.Comparing Figure 3a-b and Figure 3c-d, it is clear that lower concentration, corresponding to thinner amorphous layer, yields higher portion of vertical orientation. For $100 \mathrm{mg} / \mathrm{mL}$ concentration, the FF seed layer was dominated by bright horizontal FF stripes in random directions with a few transparent vertical domainswhich are shown as dark spots.Circulating moist air was also proved to be critical to produce a seed layer with dominant vertical domains, as shown in Figure 3c-d. The optimal seed layer with a majority of vertical domain is obtained with $50 \mathrm{mg} / \mathrm{mL}$ FF solution and circulating moist air. The highly reflective horizontal stripes become the minority in the crystallized seed layer and most of the dark silicon substrate is visible through the dense vertical FF peptidemicrorods.

This observation indicates that the crystallization process is driven by the diffusion of water molecules into the amorphousFF peptide layer. Fick's first law of diffusion suggests that the diffusion flux of water molecules in the vertical direction is proportional to the gradient of the concentration of water molecules, which in turn is proportional to the water vapor concentration at the surface and inversely proportional to the layer thickness. When a thick amorphous FF peptide layer is used, the water molecule diffusion normal to the substrate is limited and horizontal FF peptide strips are preferentially formed. In comparison, the much enhanced vertical diffusion in a thin amorphous layer with continuous supply of water molecules from circulating moist air significantly facilitates the creation of vertical FF peptidemicrorods. The Scanning Electron Microscope (SEM) imagesin Figure 4a and b show a vertical area in the seed layer, which is approximately a hexagon and consistent with its crystal structure and orientation.The zoom-in image in Figure 4b confirms this vertical seed is solid, and the scattered nanowires implythat the top surface has active sites for the self-assemble of FF molecules. The vertical seed particles are expected to initiate epitaxial growth of FF peptide crystals. AnFF peptide microrod grown from the seed layer in Figure 3dis shown in Figure 4c, along with the top view of the micro rod tip in Figure 4d and the side wall with vertical strips in Figure 4e.The rough tip and smooth side wall of the micro rod is a result of the fast growth along the axial direction and consistent with the observation that there is no branch formed.

The highly-textured seed layer and the vertically oriented microstructures enable us to conveniently characterize their piezoelectric property using PFM, which has proved challenging 
to performon free standing FF peptide nanotubes[13].The amplitude responses of the PFM measurement on the peptide seed and on the FF peptide microrod are shown in Figure 5a. The amplitude increases linearly with the applied voltage, and the slope gives the effective piezoelectric coefficient $d_{33}=9.4 \mathrm{pm} / \mathrm{V}$ for the FF peptide seed and $d_{33}=9.9 \mathrm{pm} / \mathrm{V}$ for the $\mathrm{FF}$ peptide microrod.This result indicates that the longitudinal piezoelectric coefficient $d_{33}$ of FF peptide microrods is comparable to that of $\mathrm{ZnO}$ nanowires[19], and the vertically aligned array of FF peptide microrods can potentially be a good candidate for mechanical energy conversion applications.

The PFM phase response shows that the domain pattern of the microrod is determined by the seed layer. A seed layer that had domains with random polarization resulted in the growth of microrods with random polarization, while an array of microrods with uniform polarization was achieved from a seed layer with uniform polarization. The control of the polarization was achieved using the electric field applied during the growth, while the electrical field did not affect the alignment of the microrods from this novel growth process. Whena seed layer was formed in the absence of an electric field,the PFM phase image of the seed layer in Figure $5 \mathrm{~b}$ shows many domains with opposite polarization.These antiparallel dipoles are still mostly vertical instead of randomly pointed due to the structural vertical alignment of the micro rod. The phase histogram in Figure 5d shows that the seed layer has roughly equal amount of domainswhich are $180^{\circ}$ out of phase. The microrods from such seed layer showed similar domain distribution. The piezoelectric effects of individual domains will cancel out and the microrod array will not exhibit macroscopic piezoelectric effect. Considering the difficulty in the polarization switching of FF peptide by an electric field[13, 14], which is also demonstrated in Figure S3f, we explored a novel approach for the fabrication of microrods withuniform spontaneous polarization. The uniformpolarization can be achieved through applyingan electric field during the growth. In the setup in Figure $1 \mathrm{~b}$ the applied voltagecan be switched from $0 \mathrm{~V}$ to $5 \mathrm{kV}$, which produced an electric field of $1 \mathrm{MV} / \mathrm{m}$ between a5mm electrode gap. The resultant seed layer showed much more uniform phase mapswith much less domains with the opposite phase, as shown in Figure 5c. The phase histogram in Figure 5e shows that the seed layer was dominated by domains with one polarization direction. Preliminary data indicates that most examined microrods from this seed layer were approximately in phase, but more statistical characterization for the array is needed and being investigated. 


\section{Conclusion}

To summarize, in this paper we demonstrated the low temperature fabrication of densely packed, vertically aligned FF peptide microrods. The crystal structure of FF peptide microrods with hexagonally arranged nanochannels was confirmed with XRD.The effective longitudinal piezoelectric coefficient $d_{33}$ of $9.9 \mathrm{pm} / \mathrm{V}$ was measured with PFM.Uniform spontaneous polarization was achieved by applying an electric field during the FF peptide growth. The high qualitypiezoelectric biomaterial from this work provides a new platform for applications in sensing, energy harvesting, and medicine.

\section{Acknowledgements}

The authors are truly grateful for the financial support from the Department of Mechanical Engineering and the College of Science and Engineering of the University of Minnesota. Research is also supported by in part by NSF (ECCS-1150147)and by NSF IGERT grant DGE1069104. The electron microscopy image was obtained in the Characterization Facility, University of Minnesota, which receives partial support from NSF through the MRSEC program. The device fabrication was performed in the Minnesota Nano Center, a part of the NSF-funded National Nanotechnology Infrastructure Network. 


\section{Figure Captions}

Figure 1 Schematic illustration of the fabrication process (a-c) and SEM imagesand model of FF peptidemicrorods(d-f). (a) Formation of an amorphous FF peptide layer on the substrate. (b) Formation of the FF peptide seed layer through the crystallization of the amorphous layer from (a). (c) Self-assembly of FF peptide molecules for epitaxial growth of FF peptidemicrorods. Side SEM image of the seed layer (d) and FF peptidemicrorods grown in the absence of electric field. (f) Illustration of FF peptide microrods with hexagonally arranged nanochannels. The enlarged circle illustrates a nanochannel enclosed by six FF peptide molecules.

Figure 2 X-ray powder diffraction patterns of FF peptide seeds and FF peptidemicrorods. The spectrum of FF peptide microrod is shifted upward for clarity.

Figure 3Optical images of the crystallineseed layer formed from different FF concentrations and moist air. (a) From 100mg/mL FF solution under stationary moist air. (b) From 100mg/mL FF solution under circulating moist air. (c) From 50mg/mL FF solution under stationary moist air. (d) From 50mg/mL FF solution under circulating moist air. All the amorphous layers used to form the crystalline seed layer were obtained by dropping $25 \mu \mathrm{L}$ of $\mathrm{FF}$ solution on a1.25×1.25cm square substrate.

Figure 4 (a) Top-view SEM image of a solid FF peptideseed witha roughly hexagonal shape. (b) Magnified SEM image from the circled area in a) showing nanowires leading the later growth of a FF peptide microrod. (c) Side-view SEM image of a FF peptidemicrorod. (d)Top viewmagnified image of the tip of a microrod and (e) side view magnified image of the sidewallof the micro rod in (c).

Figure 5(a) PFM amplitude response of anFF peptide seed layer and FF peptidemicrorods. Phase map of anFF peptideseed layer grown (b) without electric field and (c) with electric field by turning on the voltage in Figure 1b. (e)Histogram calculated from the phase map in (b). (f) Histogram calculated from the phase map in (c). 


\section{References}

[1] N. Santhanamoorthi, P. Kolandaivel, L. Adler-Abramovich, E. Gazit, S. Filipek, S.

Viswanathan, A. Strzelczyk, V. Renugopalakrishnan, Adv. Mater. Lett, 2 (2011) 100.

[2] R.F. Silva, D.R. Araujo, E.R. Silva, R.A. Ando, W.A. Alves, Langmuir, 29 (2013) 1020510212.

[3] X. Yan, P. Zhu, J. Li, Chem. Soc. Rev., 39 (2010) 1877-1890.

[4] J. Zhang, X. Wu, Z. Gan, X. Zhu, Y. Jin, Nano Research, 7 (2014) 929-937.

[5] L. Adler-Abramovich, D. Aronov, P. Beker, M. Yevnin, S. Stempler, L. Buzhansky, G.

Rosenman, E. Gazit, Nature Nanotechnology, 4 (2009) 849-854.

[6] P. Beker, G. Rosenman, J. Mater. Res., 25 (2010) 1661-1666.

[7] E. Bosne, A. Heredia, S. Kopyl, D. Karpinsky, A. Pinto, A. Kholkin, Appl. Phys. Lett., 102 (2013) 073504.

[8] X. Yan, J. Li, H. Möhwald, Adv. Mater., 23 (2011) 2796-2801.

[9] Q. Li, Y. Jia, L.R. Dai, Y. Yang, J.B. Li, Acs Nano, 9 (2015) 2689-2695.

[10] M. Reches, E. Gazit, Science, 300 (2003) 625-627.

[11] C.H. Görbitz, Chem. Commun., (2006) 2332-2334.

[12] M. Wang, L. Du, X. Wu, S. Xiong, P.K. Chu, Acs Nano, 5 (2011) 4448-4454.

[13] A. Kholkin, N. Amdursky, I. Bdikin, E. Gazit, G. Rosenman, Acs Nano, 4 (2010) 610-614.

[14] I. Bdikin, V. Bystrov, I. Delgadillo, J. Gracio, S. Kopyl, M. Wojtas, E. Mishina, A. Sigov, A.L. Kholkin, J. Appl. Phys., 111 (2012).

[15] A. Heredia, I. Bdikin, S. Kopyl, E. Mishina, S. Semin, A. Sigov, K. German, V. Bystrov, J. Gracio, A.L. Kholkin, Journal of Physics D-Applied Physics, 43 (2010).

[16] B. Bank-Srour, P. Becker, L. Krasovitsky, A. Gladkikh, Y. Rosenberg, Z. Barkay, G. Rosenman, Polym. J., 45 (2013) 494-503.

[17] A. Heredia, I. Bdikin, S. Kopyl, E. Mishina, S. Semin, A. Sigov, K. German, V. Bystrov, J. Gracio, A. Kholkin, J. Phys. D: Appl. Phys., 43 (2010) 462001.

[18] J. Ryu, C.B. Park, Adv. Mater., 20 (2008) 3754-3758.

[19] Z.L. Wang, J. Song, Science, 312 (2006) 242-246.

[20] J. Ryu, C.B. Park, Chem. Mater., 20 (2008) 4284-4290.

[21] C.H. Görbitz, Chemistry - A European Journal, 7 (2001) 5153-5159.

[22] X.H. Yan, J.B. Li, H. Mowald, Adv. Mater., 23 (2011) 2796-2801. 

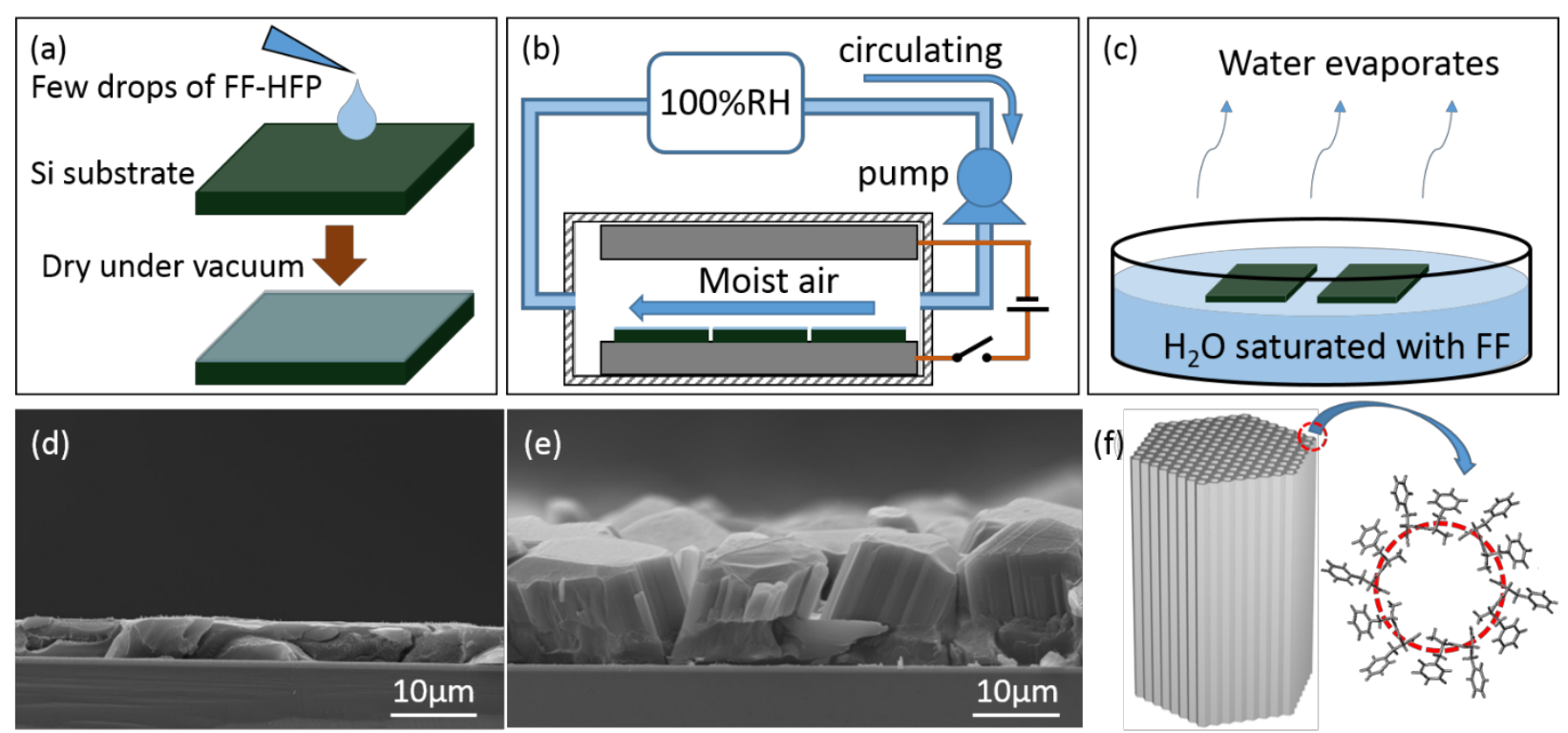

Figure 1 


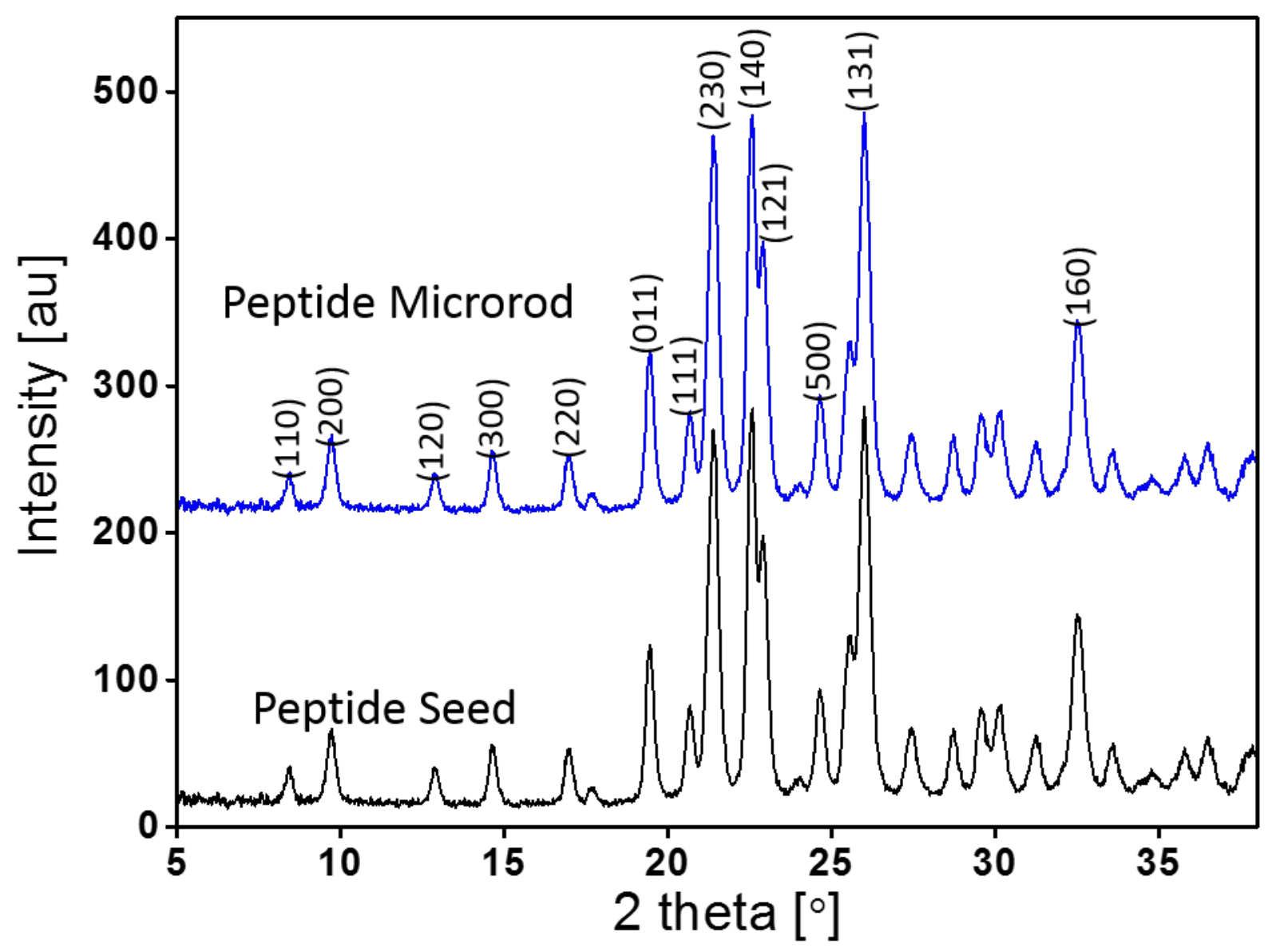

Figure 2 

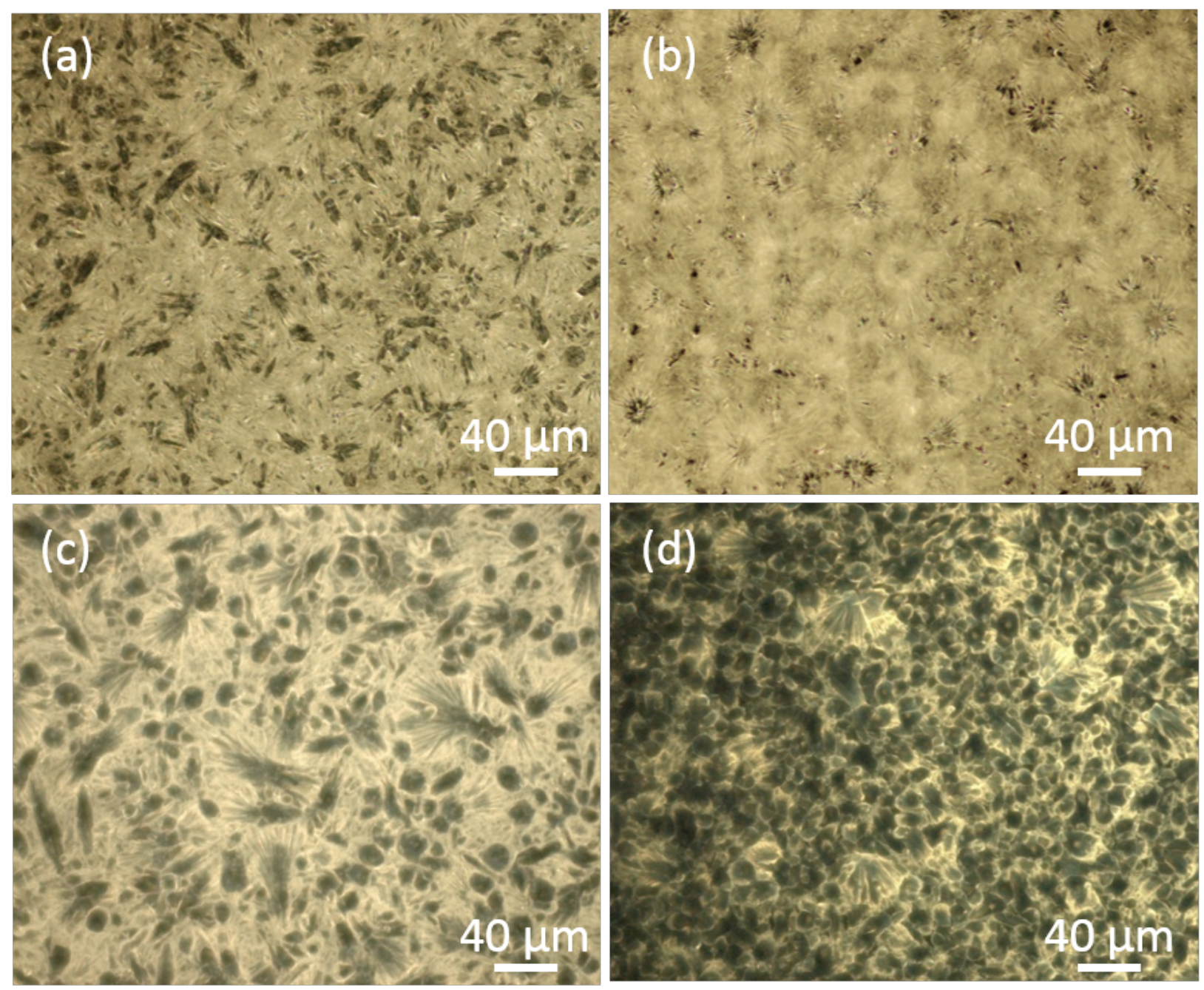

Figure 3 


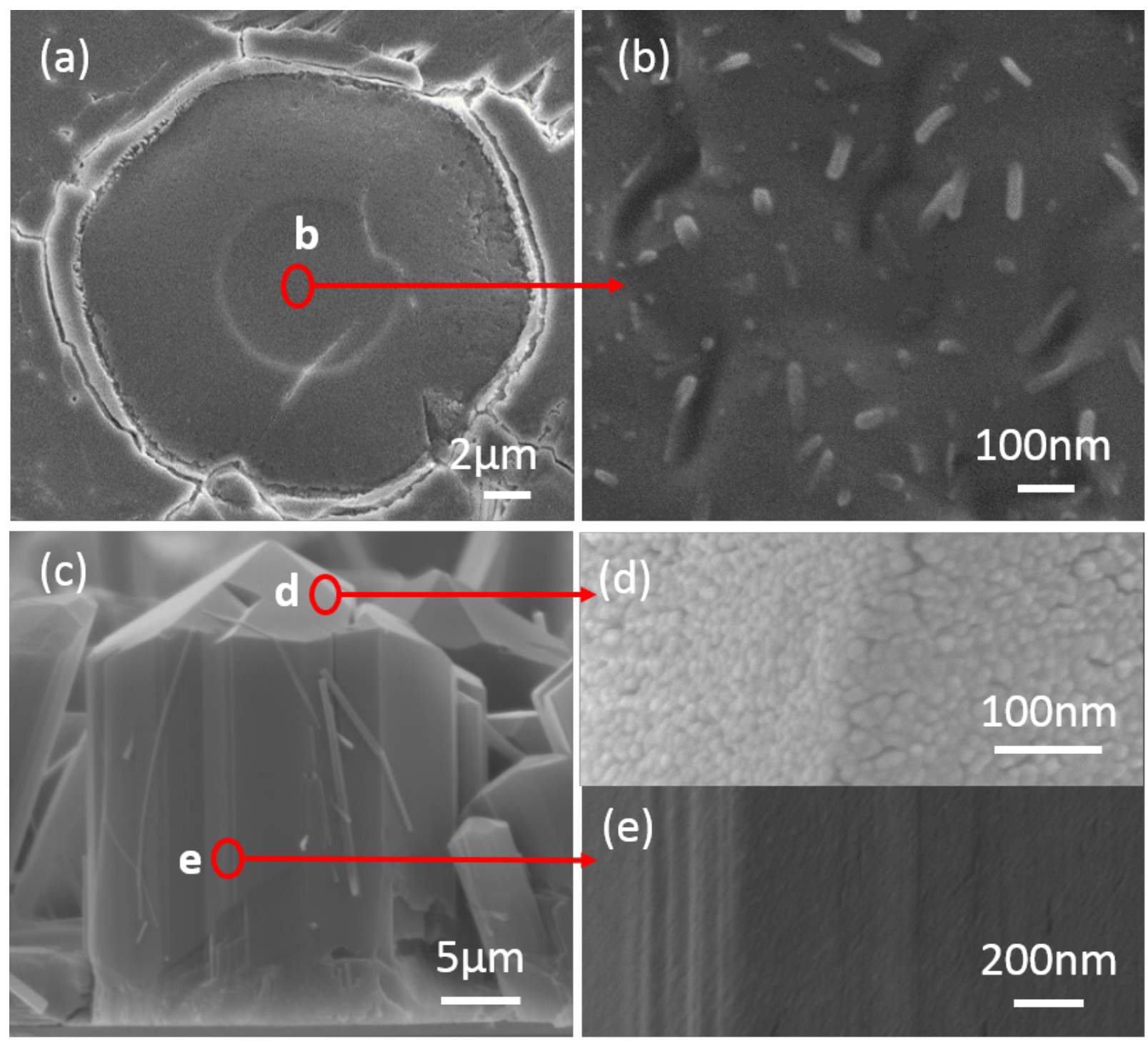

Figure 4 

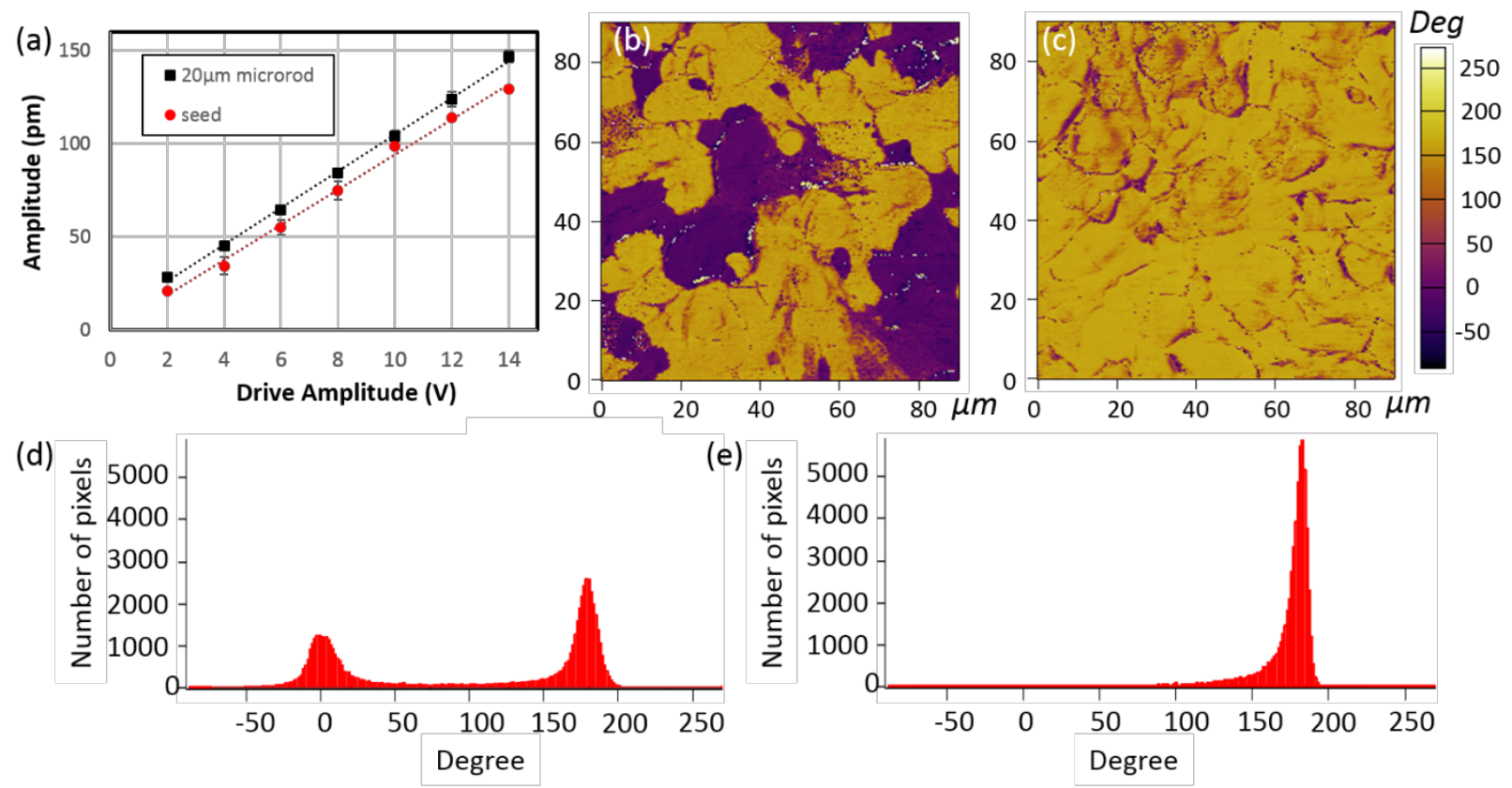

Figure 5 


\section{Vitae}

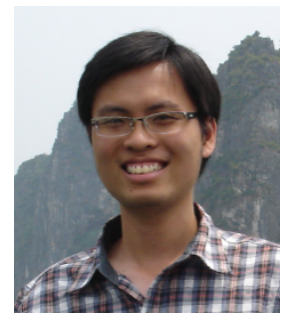

Vu Nguyen received his B.S. degree in Mechanical Engineering from Worcester Polytechnic Institute, Worcester, Massachusetts in 2012. He is currently pursuing Ph.D. degree at the University of Minnesota, Minneapolis, Minnesota. His research interests are energy harvesting and self-power systems at micro/nano scale.

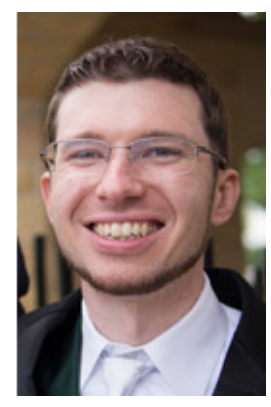

Kory Jenkins received his Bachelor's degree in Aerospace Engineering and Mechanics from the University of Minnesota in 2007. He is currently a PhD candidate at the University of Minnesota. His research interests include fabrication of novel piezoelectric sensors for haptics and human machine interaction.

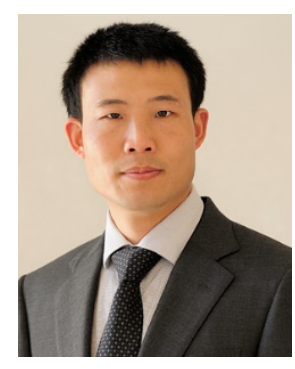

Rusen Yang received his PhD degree in Materials Science and Engineering from Georgia Institute of Technology in 2007, where he continued as Post-Doctoral Associate. He joined Mechanical Engineering at the University of Minnesota-Twin Cities as an assistant professor in 2010. He has discovered novel nanostructures, such as $\mathrm{ZnO}, \mathrm{SnO}_{2}, \mathrm{Zn}_{3} \mathrm{P}_{2}$, and investigated their 
application potentials. His most recent work on energy harvester based on piezoelectric nanomaterials made significant contribution in the field of renewable energy. 
